\title{
Propiedades ópticas de impurezas donadoras en anillos cuánticos sometidos a los efectos combinados de campo eléctrico y radiación láser intensa no resonante
}

\author{
Carlos Alberto Duque ${ }^{1, *}$, Álvaro Morales ${ }^{1}$, Miguel Mora-Ramos² \\ ${ }^{1}$ Grupo de Materia Condensada-UdeA, Instituto de Física, Facultad de Ciencias Exactas y Naturales, \\ Universidad de Antioquia UdeA, Medellín, Colombia \\ ${ }^{2}$ Facultad de Ciencias, Universidad Autónoma del Estado de Morelos, Cuernavaca, Morelos, México
}

\begin{abstract}
Resumen
Este trabajo corresponde a un estudio teórico de las propiedades ópticas y de impurezas para un electrón confinado en un anillo cuántico de GaAs-(Ga,Al)As sometido a los efectos combinados de campo eléctrico estacionario y radiación láser de alta intensidad no resonante. Los cálculos se hacen en la aproximación de masa efectiva con un proceso de diagonalización para resolver la ecuación de autovalores del Hamiltoniano. Se estudian la absorción óptica y los cambios en el índice de refracción. Los resultados obtenidos sugieren un corrimiento al rojo de las propiedades ópticas en función del campo eléctrico mientras que en el láser no resonante puede inducir efectos combinados de corrimientos al rojo y al azul. El trabajo es útil para entender las propiedades de impurezas en anillos cuánticos sometidos a perturbaciones que rompen la simetría azimutal del sistema tales como campos eléctricos estacionarios y radiación láser no resonante de alta intensidad.
\end{abstract}

Palabras clave: anillos cuánticos, impureza donadora, propiedades ópticas, campo eléctrico.

Optical properties of donor impurities in quantum rings under the combined effects of electric field and nonresonant intense laser radiation

\begin{abstract}
This work corresponds to a theoretical study of the optical properties of impurities for an electron confined in a GaAs- $(\mathrm{Ga}, \mathrm{Al})$ As quantum ring under the combined effects of stationary electric field and high intensity nonresonant laser radiation. Calculations are made on the effective mass approximation with a diagonalization process to solve the eigenvalues equation of the Hamiltonian. The optical absorption and the changes in refractive index are studied. The results suggest a redshift of the optical properties as a function of the electric field while the nonresonant laser can induce combined effects of redshifts and blueshifts. The work is useful for understanding the properties of impurities in quantum rings subjected to perturbations which break the azimuthal symmetry of the system such as non-stationary resonant electric fields and high-intensity laser radiation.
\end{abstract}

Key words: Quantum rings, donor impurity, optical properties, electric field.

\section{Introducción}

El reciente progreso en la tecnología de nanofabricación ha hecho posible el diseño y la producción de nuevas estructuras y nuevos dispositivos sobre la base de materiales semiconductores y dieléctricos, en los cuales es posible observar una importante variedad de fenómenos físicos novedosos. Entre ellos se pueden mencionar, la observación de estados moleculares en puntos cuánticos espacialmente acoplados (Bayear, et al., 2001) y la formación de anillos cuánticos de tamaño nanométrico (García, et al., 1997), que son análogos nanoscópicos del benceno. En particular, la fabricación de anillos cuánticos semiconductores ha despertado gran interés por la realización de los fenómenos cuánticos topológicos, que se espera se manifiesten en sistemas de geometría reducida (Aharonov \& Bohm, 1959). Por ejemplo, Lorke, et al. observaron por primera vez la respuesta óptica de infrarrojo lejano en anillos cuánticos autoensamblados; revelando cambios en el estado fundamental inducidos magnéticamente (Lorke, et al., 2000).

Por otro lado, también se ha estudiado la influencia de campos eléctricos laterales sobre las propiedades electrónicas y ópticas de anillos cuánticos en el régimen de confinamiento fuerte (Harutyunyan, 2009; Harutyunyan, 2011). Cabe mencionar que varios de esos estudios han investigado los

\footnotetext{
*Correspondencia:

Carlos Alberto Duque, cduque_echeverri@yahoo.es

Recibido: 01 de diciembre de 2014

Aceptado: 14 de mayo de 2015
} 
estados excitónicos; así como las variaciones que se pueden inducir en el espectro de electroabsorción excitónica. En otro orden de cosas, Monozon y Schmelcher reportaron un conjunto de aproximaciones analíticas al problema de un electrón sometido al efecto de una impureza en anillos cuánticos en presencia de campos eléctricos y magnéticos (Monozon \& Schmelcher, 2003) , en tanto Aichinger y colaboradores consideraron los efectos de impurezas y las variaciones de la geometría sobre los niveles de muchos electrones confinados en anillos cuánticos sometidos a campos magnéticos externos (Aichinger, et al., 2006).

En los últimos años se han publicado una gran variedad de trabajos que tienen como objeto de estudio heteroestructuras en las cuáles se pueden encontrar ciertas simetrías que se preservan. Esto ocurre ya sea por la configuración específica del sistema o por la forma en que se aplican diversas perturbaciones externas. En los anillos cuánticos, en particular, se preserva la simetría azimutal (Filikhin, et al., 2006; Culchac, et al., 2008; Bruno-Alfonso \& Latgé, 2000; Assaid, et al., 2008; Kleemans, et al., 2009). Muchos de los trabajos teóricos acerca de estos sistemas se han desarrollado haciendo uso de la aproximación de masa efectiva, conjuntamente con el empleo de esquemas de cálculo de distinta naturaleza: variacionales, perturbativos y, más recientemente, mediante el desarrollo de las funciones de onda en términos de conjuntos completos que constituyen bases adecuadas del espacio de Hilbert. Todo esto apuntando al objetivo central que es encontrar las autofunciones y autoenergías del Hamiltoniano del sistema. Tales cálculos han sido desarrollados para investigar distintas clases de problemas, como son: (1) espectros de electrones y huecos en anillos cuánticos aislados (Filikhin, et al., 2006) y dobles (Culchac, et al., 2008); (2) estados de impurezas aceptoras y donadoras poco profundas en anillos cuánticos con confinamiento rectangular bajo los efectos cruzados de campos eléctrico y magnético (Bruno-Alfonso \& Latgé, 2000); (3) estados de impurezas poco profundas en puntos cuánticos parabólicos y semiparabólicos (Assaid, et al., 2008), y (4) complejos excitónicos en puntos cuánticos verticalmente acoplados bajo los efectos de campo magnético (Kleemans, et al., 2009). Los resultados más importantes pueden ser resumidos así: (1) los niveles excitónicos no aparecen equidistantes y se desdoblan con el campo magnético, reflejando con ello las características asociadas a la geometría de los anillos, (2) las oscilaciones de Aharonov-Bohm de excitones, que son características de anillos unidimensionales no aparecen en sistemas de dimensiones finitas, y (3) los campos eléctricos generan nuevas reglas de selección para las transiciones inter e intrabanda.

Estudios recientes han reportado el coeficiente de absorción óptica intrabanda lineal y no lineal en anillos cuánticos de GaAs aislados bajo los efectos de presión hidrostática y campo eléctrico (Barseghyan, et al., 2012). Entre los resultados arrojados en dicha investigación está que la variación de los diferentes tamaños característicos de la geometría de la estructura puede generar corrimientos bien sea al rojo o al azul de los picos resonantes del espectro óptico intrabanda. Adicionalmente, se ha demostrado que la presencia de un campo eléctrico estático conduce a un desplazamiento hacia el rojo, mientras que la influencia de la presión hidrostática conduce a un desplazamiento hacia el azul en los espectros. En la misma línea de la óptica no lineal, algunos trabajos han reportado los estados de energía en un complejo $\mathrm{D}_{2}{ }^{+}$formado por el acoplamiento de un electrón en la banda de conducción y dos centros donadores en un anillo cuántico con sección transversal rectangular, destacando la importante contribución de la interacción repulsiva entre los dos centros de Coulomb (Fulla, et al., 2014). En ese reporte se discuten también las condiciones que conducen a la destrucción de las oscilaciones Aharonov-Bohm del estado fundamental.

Teniendo en cuenta que las perturbaciones externas aplicadas son herramientas útiles para cambiar las propiedades electrónicas y ópticas de estructuras semiconductoras, se han potenciado nuevos estudios que permiten demostrar teóricamente que las nanoestructuras irradiadas por un campo de láser intenso (ILF del Inglés) presentan unas características muy diferentes a las de un semiconductor en volumen y que los efectos de la radiación se hacen mucho más pronunciados a medida que se incrementa el confinamiento de los portadores dada la reducción en la dimensionalidad de los sistemas (Duque, et al., 2011; Mora-Ramos, et al., 2013). Así, Peyghambarian et al. reportaron por vez primera la observación del efecto Stark sobre excitones sometidos a un ILF no resonante (Peyghambarian, et al., 1989), mientras Asmar y colaboradores consideraron un gas de electrones cuasi bidimensional en heteroestructuras de GaAs vestidas por un campo láser intenso de frecuencias en el rango de los terahertz (Asmar, et al., 1995). En la actualidad existe gran desconocimiento sobre la alta capacidad de ajuste de las transiciones inter-subbanda en sistemas unidimensionales y bidimensionales bajo los efectos de láser intenso no resonante. También se desconocen los efectos de la aplicación combinada de radiación de láser intenso y campos eléctricos estacionarios aplicados en el plano de heteroestructuras cuasi-bidimensionales. Los efectos de una radiación ILF en el rango de los terehartz sobre el potencial de confinamiento de puntos y anillos cuánticos pueden ser importantes para el diseño de nuevos láseres sintonizables, moduladores ópticos y detectores de infrarrojos ultra-rápidos.

El presente estudio está dedicado a la modelación de una impureza donadora poco profunda confinada en un anillo cuántico que está sometido a los efectos combinados de un campo eléctrico estacionario y a una radiación ILF no resonante polarizada en el plano del anillo. El campo eléctrico estacionario y la polarización de la radiación son paralelos. Los cálculos se hacen usando las aproximaciones de masa efectiva y bandas parabólicas. En el proceso de 
obtener las funciones de onda y energías se hace uso de un esquema de desarrollo de la función de onda en una base de funciones sinusoidales asociadas a un electrón confinado en una región cuadrada de ancho suficientemente grande y que está restringida por un potencial de confinamiento infinito.

El artículo está organizado de la siguiente manera. En la sección 2 se describe el marco teórico. La sección 3 presenta los resultados y discusión de los mismos. Por último, las conclusiones se resumen en la Sección 4.

\section{Marco teórico}

El sistema bajo estudio consiste de un electrón confinado en un anillo cuántico de GaAs de radios $R_{1}<R_{2}$, rodeado por una matriz de $\mathrm{Ga}_{1-\mathrm{x}} \mathrm{Al}_{\mathrm{x}} \mathrm{As}$, con el origen de coordenadas localizado en el centro del anillo. Se implanta una impureza donadora en las coordenadas $\left(x_{0}, y_{0}\right)$ y se aplica un campo eléctrico en dirección negativa del eje $x$. El Hamiltoniano para dicho sistema, en coordenadas cartesianas, está dado por:

$$
-\frac{\hbar^{2}}{2 m^{*}}\left(\frac{\partial^{2}}{\partial x^{2}}+\frac{\partial^{2}}{\partial y^{2}}\right)+V(x, y)-\frac{e^{2}}{\epsilon_{r} r}+e F x,
$$

donde $m^{*}$ y $e$ son la masa efectiva y la carga del electrón, respectivamente, $V(x, y)$ es el potencial de confinamiento asociado al anillo cuántico, $\epsilon_{r}$ es la constante dieléctrica del GaAs, $F$ es la magnitud del campo eléctrico aplicado y $\mathrm{r}=\sqrt{\left(x-x_{0}\right)^{2}+\left(y-y_{0}\right)^{2}}$ es la distancia entre la impureza y electrón [el cual está localizado en el punto de coordenadas $(x, y)]$. En el presente modelo el potencial de confinamiento es cero en la región del anillo $\left(R_{1} \leq r \leq R_{2}\right)$ y $V(x, y)=V_{0}$ en las demás regiones.

Para introducir los efectos de láser se sigue el modelo de un electrón en un campo de radiación intensa [ver detalles en las Refs. (Duque, et al., 2010; Kasapoglu, et al., 2011)]. Esencialmente, el problema se aproxima a considerar la ecuación 1 con un potencial de confinamiento "vestido" por el efecto del láser. En ese caso se hace el cambio $V(x, y) \rightarrow \mathcal{V}\left(x, \mathrm{y}, \alpha_{0}\right)$, donde (Radu, et al., 2014)

$$
\begin{aligned}
\mathcal{V}\left(x, \mathrm{y}, \alpha_{0}\right)= & \frac{V_{0}}{\pi} \operatorname{Re}\left[\pi-\Theta\left(\alpha_{0}-x-\operatorname{Re}\left(\mathrm{z}_{1}\right)\right) \cos ^{-1}\left(\frac{\operatorname{Re}\left(\mathrm{z}_{1}\right)+x}{\alpha_{0}}\right)\right. \\
& +\Theta\left(\alpha_{0}-x-\operatorname{Re}\left(\mathrm{z}_{2}\right)\right) \cos ^{-1}\left(\frac{\operatorname{Re}\left(\mathrm{z}_{2}\right)+x}{\alpha_{0}}\right) \\
& -\Theta\left(\alpha_{0}-x-\operatorname{Re}\left(\mathrm{z}_{1}\right)\right) \cos ^{-1}\left(\frac{\operatorname{Re}\left(\mathrm{z}_{1}\right)+x}{\alpha_{0}}\right) \\
& +\Theta\left(\alpha_{0}-x-\operatorname{Re}\left(\mathrm{z}_{2}\right)\right) \cos ^{-1}\left(\frac{\operatorname{Re}\left(\mathrm{z}_{2}\right)+x}{\alpha_{0}}\right)
\end{aligned}
$$

En la ecuación (2), $z_{1}=\sqrt{R_{1}^{2}-y^{2}}, z_{2}=\sqrt{R_{2}^{2}-y^{2}}$. Además, $\Theta(w)$ es la función de paso unitario de Heaviside y $\operatorname{Re}(Z)$ significa la parte real del número complejo $Z$. Aquí, la cantidad $\alpha_{0}\left(=e A_{0} /\left(c m^{*} \omega_{d}\right)\right.$ es el parámetro asociado a la radiación láser incidente (de frecuencia $\omega_{d}$ ), $c$ es la velocidad de la luz y $A_{0}$ es una constante, la cuál ha sido elegida con polarización lineal en dirección $x$.

Para hallar las autofunciones y autoenergías del operador de Hamilton en la ecuación (1), se puede proceder, por ejemplo, siguiendo un método de diagonalización donde se haga uso de un conjunto completo de funciones ortonormales mediante las cuales se pueda hacer un desarrollo de la función de onda del electrón confinado en la región de estudio y que está sometido a las diferentes perturbaciones que han sido consideradas sobre el sistema. Tal enfoque conduce a la construcción de una matriz hamiltoniana, a la cual se le buscan sus autovalores y autovectores, que no son otra cosa que las energías de los estados cuánticos posibles y los conjuntos de coeficientes del desarrollo para cada estado, respectivamente. En el presente estudio se ha usado como base un conjunto de funciones sinusoidales que son autofunciones del Hamiltoniano para un electrón confinado en una caja cuadrada de lados $L_{x}$ x $L_{y}$ con potencial de confinamiento infinito [ver detalles en Ref. (Xia \& Fan, 1989)]. Una vez obtenidas las funciones de onda y las autoenergías se puede proceder a calcular por ejemplo las propiedades ópticas lineales y no lineales tales como absorción óptica y cambios en el índice de refracción.

Usando la técnica de matriz densidad y expresando la polarización eléctrica en una serie de potencias del campo eléctrico de la radiación incidente, se puede proceder a calcular la parte lineal y la corrección de tercer orden del coeficiente de absorción óptica. Respectivamente, ambas contribuciones están dadas por [ver detalles en la Ref. (Martínez-Orozco, et al., 2012)]:

$$
\begin{gathered}
\alpha^{1}(\omega)=\omega \sqrt{\frac{\mu_{0}}{\epsilon_{0} \epsilon_{r}}}\left[\frac{e^{2} \sigma \hbar \Gamma_{i f}\left|M_{i f}\right|^{2}}{\left(E_{i f}^{\omega}\right)^{2}+\left(\hbar \Gamma_{i f}\right)^{2}}\right] \\
\alpha^{3}(\omega, I)=-\omega \sqrt{\frac{\mu_{0}}{\epsilon_{0} \epsilon_{r}}}\left(\frac{e^{4} I}{2 n \epsilon_{0} c}\right)\left\{\frac{\sigma \hbar \Gamma_{i f}\left|M_{i f}\right|^{2}}{\left[\left(E_{i f}^{\omega}\right)^{2}+\left(\hbar \Gamma_{i f}\right)^{2}\right]^{2}}\right\} \\
\times\left\{4\left|M_{i f}\right|^{2}\right. \\
\left.-\frac{\left|M_{f f}-M_{i i}\right|^{2}\left[3 E_{i f}^{2}-4 E_{i f} \hbar \omega+\hbar^{2}\left(\omega^{2}-\Gamma_{i f}\right)\right]}{E_{i f}^{2}+\left(\hbar \Gamma_{i f}\right)^{2}}\right\}
\end{gathered}
$$

Pueden calcularse también los coeficientes lineal y no lineal (de tercer orden) del cambio en el índice de refracción, que resultan estar dados por

$$
\frac{\Delta n^{1}(\omega)}{n}=\left(\frac{e^{2} \sigma\left|M_{i f}\right|^{2}}{2 n^{2} \epsilon_{0}}\right)\left[\frac{E_{i f}^{\omega}}{\left(E_{i f}^{\omega}\right)^{2}+\left(\hbar \Gamma_{i f}\right)^{2}}\right]
$$




$$
\begin{aligned}
\frac{\Delta n^{3}(\omega)}{n}=- & \left(\frac{e^{4} \sigma\left|M_{i f}\right|^{2}}{4 n^{3} \epsilon_{0}}\right)\left\{\frac{c I \mu_{0}}{\left[\left(E_{i f}^{\omega}\right)^{2}+\left(\hbar \Gamma_{i f}\right)^{2}\right]^{2}}\right\} \\
& \times\left\{4 E_{i f}^{\omega}\left|M_{i f}\right|^{2}-\frac{\left|M_{f f}-M_{i i}\right|^{2}}{E_{i f}^{2}+\left(\hbar \Gamma_{i f}\right)^{2}}\right. \\
& \times\left\{E_{i f}^{\omega}\left[E_{i f} E_{i f}^{\omega}-\left(\hbar \Gamma_{i f}\right)^{2}\right]\right. \\
& \left.\left.-\left(\hbar \Gamma_{i f}\right)^{2}\left(E_{i f}+E_{i f}^{\omega}\right)\right\}\right\}
\end{aligned}
$$

donde $E_{i f}^{\omega}=E_{i f}-\hbar \omega, E_{i f}=E_{f}-E_{i}$ es la energía de transición inter-subbanda y $\hbar \omega$ es la energía del fotón, de frecuencia $\omega$, que genera la transición entre niveles. Por otra parte, $M_{i f}=\left\langle\Psi_{f}|x| \Psi_{i}\right\rangle$ es proporcional al momento de dipolo eléctrico de la transición entre los estados $\Psi_{f}$ y $\Psi_{i}$, en tanto que $\Gamma_{i f}$ es proporcional a la tasa de relajación entre los estados final e inicial. Adicionalmente, $\sigma$ es la densidad tridimensional de electrones, $n=\sqrt{\epsilon_{r}}$ es el índice de refracción del material (GaAs en este estudio), $\mu_{0}$ es la permeabilidad magnética del vacío, $\epsilon_{0}$ es la susceptibilidad del vacío, $I$ es la intensidad de la radiación incidente que se usa para generar la transición intersubbandas, y $c$ es la velocidad de la luz en el vacío. Una vez obtenidos los términos de primer y tercer orden la absorción total es el resultado de hacer la suma de dichas contribuciones. Igual procedimiento se sigue para calcular los cambios totales al índice de refracción.

\section{Resultados y discusión}

A continuación se procede a presentar los resultados obtenidos en el presente estudio. Los parámetros usados en el cálculo son: $m^{*}=0.0665 m_{0}$ (donde $m_{0}$ es la masa del electrón libre), $L_{x}=L_{y}=70 \mathrm{~nm}, V_{0}=228 \mathrm{meV}$, (que corresponde a una concentración de aluminio de $x=0.3$ en las regiones de la barrera), $\epsilon_{r}=12.58, \sigma=3.8 \times 10^{22} \mathrm{~m}^{-3}, \quad \Gamma_{i f}$ $=5 \mathrm{THz}, I=0.2 \times 10^{10} \mathrm{~W} / \mathrm{m}^{2}$.

En la figura 1 se presenta la función de onda para los tres primeros estados de un electrón confinado en un anillo cuántico de $\mathrm{GaAs} / \mathrm{Ga}_{0.7} \mathrm{Al}_{0.3} \mathrm{As}$. De izquierda a derecha las columnas corresponden, respectivamente, al estado fundamental, primer y segundo estados excitados. En la primera fila el sistema corresponde a un electrón confinado en el anillo sin efectos de impureza y con $\alpha_{0}=0$. En las tres filas restantes la impureza está localizada en el punto de coordenadas $\left(\frac{R_{1}+R_{2}}{2}, 0\right)$ y tres valores del parámetro de laser han sido considerados $\left(\alpha_{0}=0,5 \mathrm{~nm}, 10 \mathrm{~nm}\right)$. En los gráficos de la primera fila se puede observar, por ejemplo, la simetría azimutal característica de los estados $1 s$. Adicionalmente, notamos que para dicho estado la densidad de probabilidad decrece en la medida que nos aproximamos hacia las regiones externas del anillo (tanto para $\rho<R_{1}$ como para $\rho$ $<R_{2}$ ). Esta situación se debe fundamentalmente a que, para el estado fundamental, la barrera de potencial se comporta
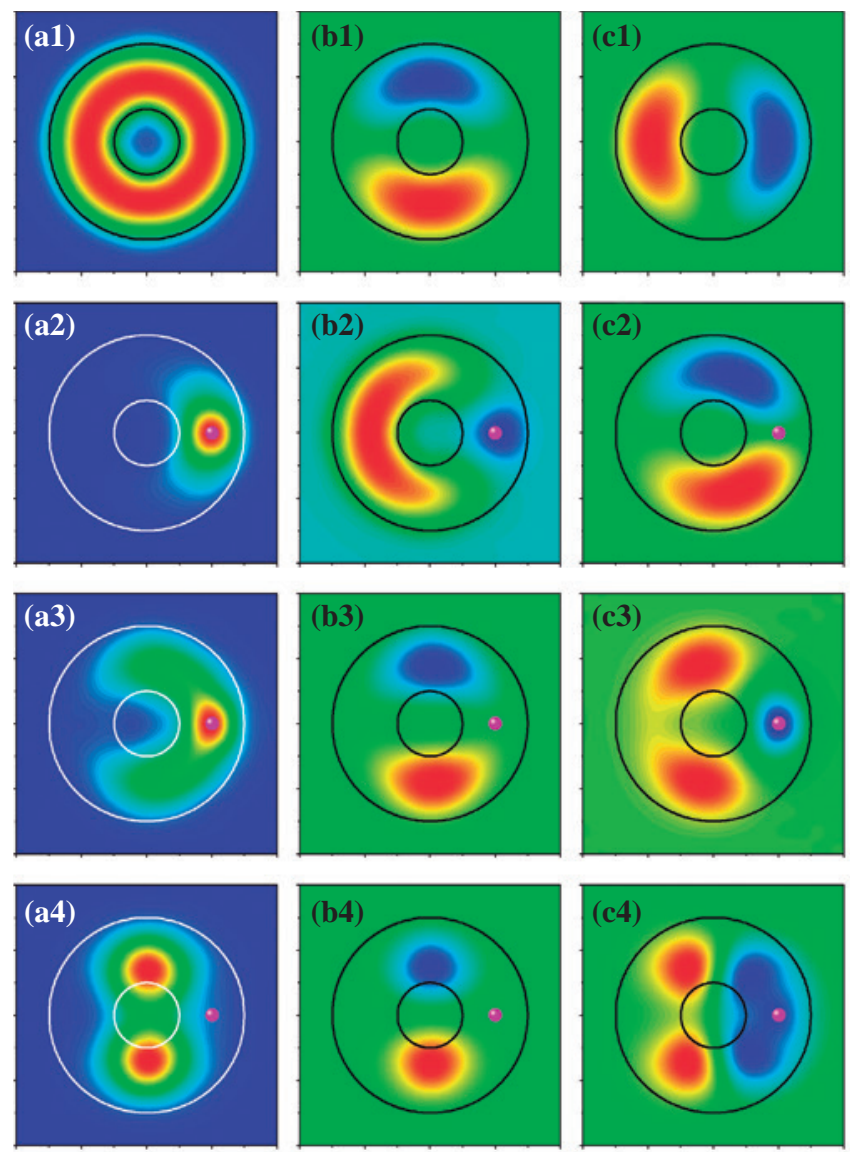

Figura 1. Función de onda para los tres primeros estados de un electrón confinado en un anillo cuántico de $\mathrm{GaAs} / \mathrm{Ga}_{0.7} \mathrm{Al}_{0.3} \mathrm{As}$ con $R_{1}$ $=5 \mathrm{~nm}, R_{2}=25 \mathrm{~nm}$ y $\alpha_{0}=5 \mathrm{~nm}$. De izquierda a derecha las columnas corresponden, respectivamente, al estado fundamental, primer y segundo estados excitados. En la primera fila el sistema corresponde a un electrón confinado en el anillo sin efectos de impureza y con $F=0$. En las tres filas restantes la impureza está localizada en el punto de coordenadas $\left(\frac{R_{1}+R_{2}}{2}, 0\right)$ (ver el círculo violeta en las tres filas inferiores) y tres valores del parámetro de láser han sido considerados. Así, desde arriba hacia abajo las filas corresponden a tres valores del parámetro de laser $\left(\alpha_{0}=0,5 \mathrm{~nm}, 10 \mathrm{~nm}\right)$.

esencialmente como una barrera de altura infinita. En el caso de los primeros dos estados excitados, se observa el comportamiento característico tipo $2 \rho_{x} 2 \rho_{y}$. Es decir, estados que son idénticamente nulos en el origen y con lóbulos marcados en direcciones de los ejes de coordenadas $x$ y $y$. La suma de las dos densidades de probabilidad asociadas a estos dos estados excitados corresponde nuevamente a una situación de simetría azimutal donde la densidad de probabilidad resultante es idénticamente igual a cero en el origen. Aquí es importante resaltar que los dos estados excitados son degenerados para la energía $\left(E_{1}=E_{2}\right)$.

Al considerar los efectos de una impureza (filas 2, 3 y 4), claramente se observa un rompimiento de la simetría azimutal. En el caso donde $\alpha_{0}=0$, el estado fundamental 
es esencialmente de simetría esférica alrededor de la impureza con pequeñas deformaciones debida a las barreras de potencial en el anillo. Asimismo, los estados excitados conservan la forma característica de estados $2 p$ con rompimientos de simetría debido a la presencia no simétrica de la impureza. Al incluir los efectos del campo de láser intenso, $\alpha_{0}=5 \mathrm{~nm}$, aparece una deslocalización del estado fundamental, con la función de onda extendida sobre el eje $y$, llegando a presentar dos antinodos bien definidos. De igual manera, se manifiesta un estado $2 p_{y}$ muy bien definido dada la presencia de una barrera central que se induce por efectos de láser. En cambio, en el caso del estado $2 p_{x}$ se observa una deslocalización de la función de onda debido a los dos pozos de potencial que se inducen a lo largo de la dirección $x$.

Claramente, la degeneración de la energía de los dos primeros estados excitados se rompe producto del cambio en la simetría inducido tanto por la presencia de la impureza como por el efecto del campo de láser, como puede apreciarse en las filas 3 y 4 de la Fig. 1.

Por otra parte, en la figura 2 se presentan los resultados para la energía de los primeros estados de un electrón en un anillo cuántico de $\mathrm{GaAs} / \mathrm{Ga}_{0.7} \mathrm{Al}_{0.3} \mathrm{As}$ en función de $\alpha_{0}$ con y sin efectos de impureza y considerando dos posiciones diferentes de la misma. En general se observa que en ausencia de impureza y sin efectos de láser aparecen las degeneraciones propias de sistemas que tienen simetría azimutal [Fig. 2(a)] y que todos los estados aquí reportados tienen una energía creciente en función de $\alpha_{0}$.
Hay dos razones que explican tal clase de comportamiento: 1) para valores pequeños de $\alpha_{0}$ se produce una reducción a lo largo de la dirección $x$ del diámetro de los círculos que forman el anillo, generando con ello un mayor confinamiento de los portadores y por tanto un incremento de la energía; y 2) para valores altos de $\alpha_{0}$ aparece un desplazamiento hacia el azul del nivel de referencia de la energía (el fondo del pozo de potencial), lo que a su vez implica un crecimiento en la energía de los portadores. En aquellas situaciones donde hay impureza y debido a que esta se encuentra ubicada en puntos de no simetría, se observa que en ausencia de efectos de láser incluso se pierde la degeneración de los estados. En presencia de impureza se observa un corrimiento al rojo de todos los estados, siendo el efecto más pronunciado sobre el estado fundamental, lo cual se asocia al carácter atractivo de la impureza que, dependiendo de las dimensiones de la estructura, puede tender a solapar los efectos del confinamiento geométrico.

El efecto de la aplicación de un campo eléctrico estacionario sobre las funciones de onda de los tres primeros estados de un electrón confinado en un anillo cuántico de GaAs/ $\mathrm{Ga}_{0.7} \mathrm{Al}_{0.3} \mathrm{As}$ con $R_{1}=5 \mathrm{~nm}, R_{2}=25 \mathrm{~nm}$ y $\alpha_{0}=5 \mathrm{~nm}$, aparece representado en la figura 3 . En este caso, las filas corresponden a diferentes configuraciones del campo eléctrico aplicado; sin la inclusión de átomo de impureza (primera fila), y con los efectos de una impureza localizada a lo largo del eje $x$. Dado que en este caso desde el principio se está considerando un campo de láser diferente de cero, está claro que el sistema de entrada no preserva la simetría

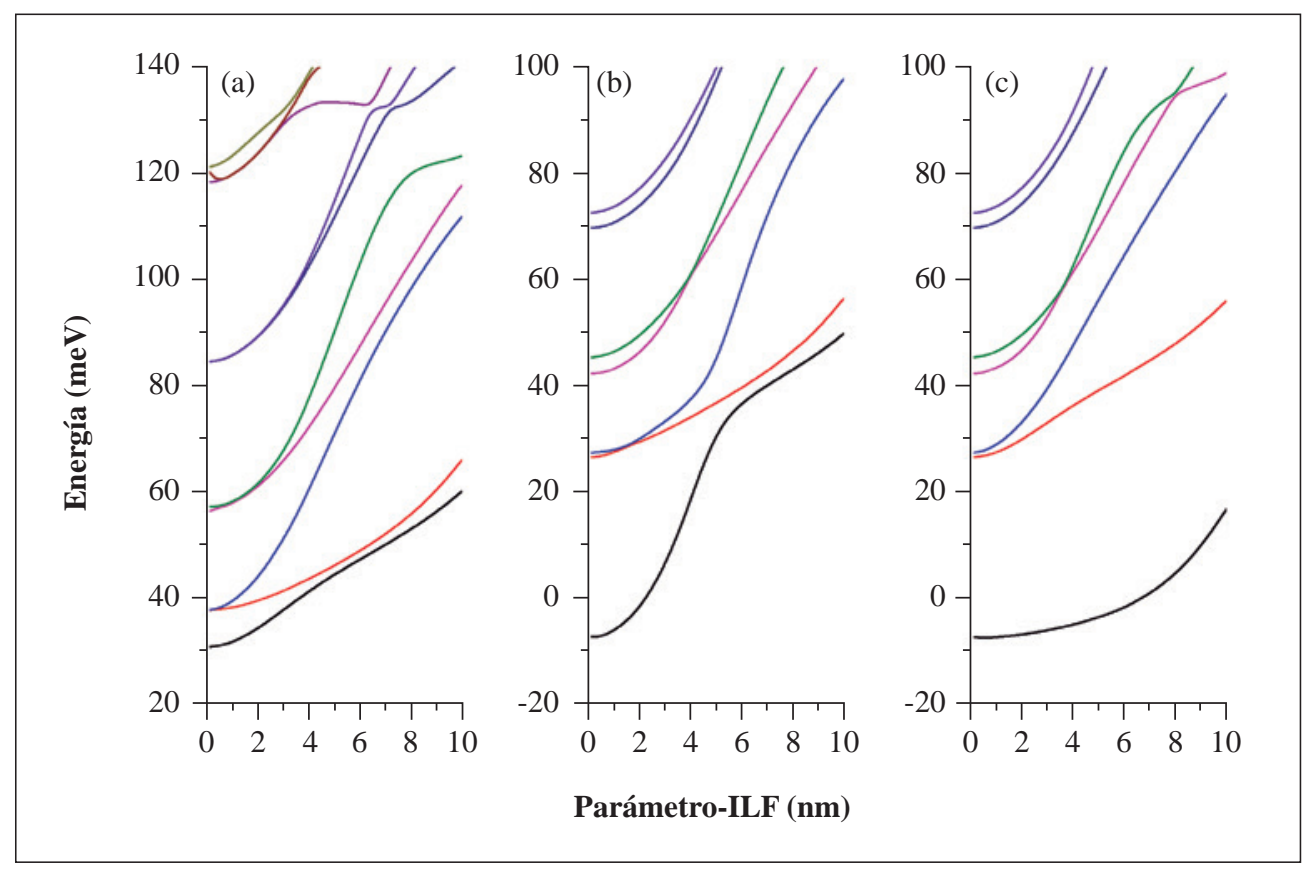

Figura 2. Energía de los primeros estados de un electrón en un anillo cuántico de GaAs/ $\mathrm{Ga}_{0.7} \mathrm{Al}_{0.3} \mathrm{As}$ en función de $\alpha_{0}$. Los resultados son para $R_{1}=5 \mathrm{~nm}, R_{2}=15 \mathrm{~nm}$ y $F=0$. En (a) no se consideran efectos de impureza mientras que en (b) y (c) la impureza está localizada, respectivamente, en las coordenadas $\left(\frac{R_{1}+R_{2}}{2}, 0\right)$ y $\left(0, \frac{R_{1}+R_{2}}{2}\right)$. 

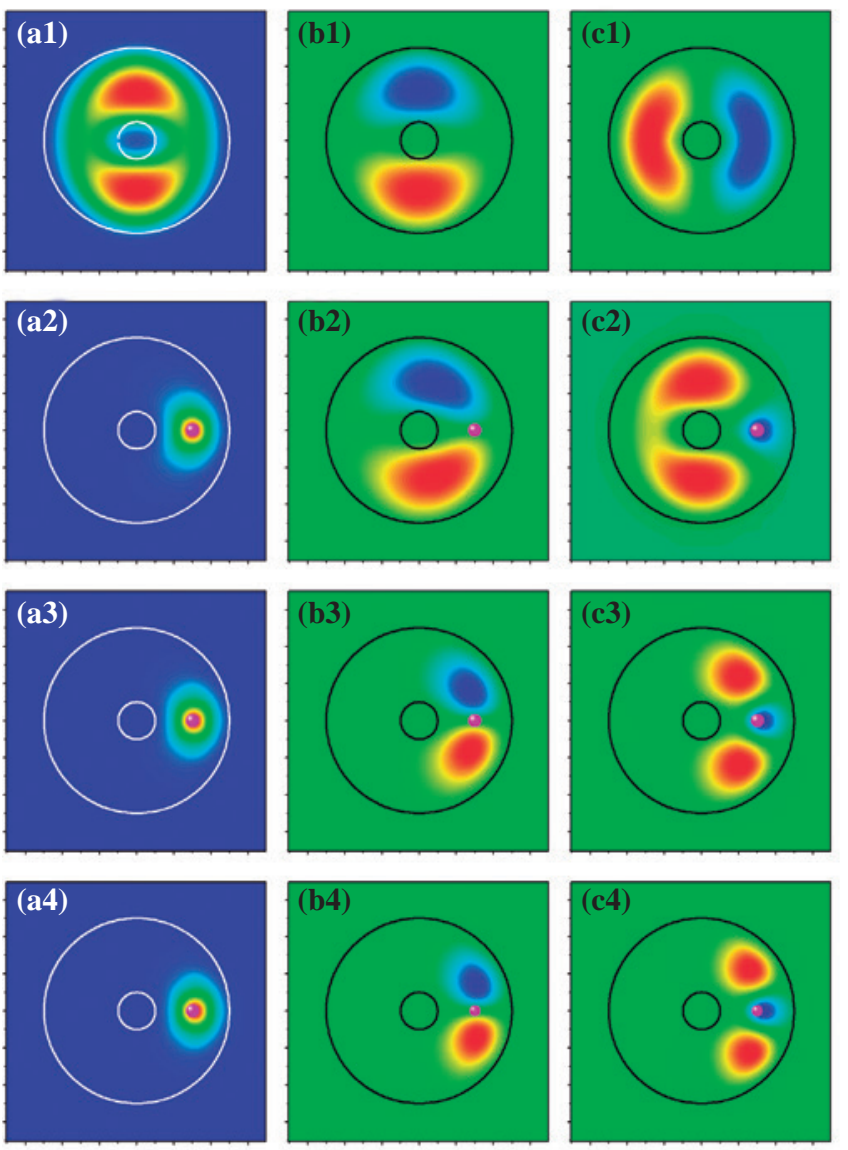

Figura 3. Función de onda para los tres primeros estados de un electrón confinado en un anillo cuántico de $\mathrm{GaAs} / \mathrm{Ga}_{0.7} \mathrm{Al}_{0.3} \mathrm{As}$ con $R_{1}=5 \mathrm{~nm}, R_{2}=25 \mathrm{~nm}$ y $\alpha_{0}=5 \mathrm{~nm}$. De izquierda a derecha las columnas corresponden, respectivamente, al estado fundamental, primer y segundo estados excitados. En la primera fila el sistema corresponde a un electrón confinado en el anillo sin efectos de impureza y con $F=0$. En las tres filas restantes la impureza está localizada en el punto de coordenadas $\left(\frac{R_{1}+R_{2}}{2}, 0\right)$ (ver el punto violeta en las tres filas inferiores) y tres valores del campo eléctrico han sido considerados De arriba hacia abajo ellos son: $(F=0,20$ $\mathrm{kV/cm}, 40 \mathrm{kV} / \mathrm{cm})$.

azimutal, tal como puede apreciarse en las funciones de onda del problema sin impureza (ver primera fila de la figura 3). El potencial de confinamiento corresponde a un disco que se desplaza a lo largo del eje $x$, teniendo una barrera central que disminuye de ancho a medida que el potencial aumenta. Este tipo de potencial, que no es abrupto, genera un mayor confinamiento sobre los estados de más baja energía y permite una mayor deslocalización de los estados de más alta energía. Al considerar los efectos de impureza -segunda fila de la figura 3-, se observa que el estado fundamental aparece fuertemente localizado alrededor de la impureza, con un carácter bastante simétrico dadas las grandes dimensiones de la sección transversal del anillo. En los paneles b2 y c2 se puede notar que la impureza desplaza hacia ella la nube electrónica, pero mostrando aún las funciones de onda el carácter $2 p$ de las mismas. Dado que en este caso el campo eléctrico se aplica en dirección negativa del eje $x$, se entiende claramente por qué en las filas 3 y 4 las funciones de onda se desplazan hacia la posición de la impureza. Esto, más adelante, explicará la forma creciente que muestra la energía de enlace a medida que crece el campo eléctrico, particularmente en el caso de la impureza a lo largo del eje $x$.

La dependencia de la energía de los primeros estados electrónicos en un anillo cuántico de $\mathrm{GaAs} / \mathrm{Ga}_{0.7} \mathrm{Al}_{0.3} \mathrm{As}$, en función del campo eléctrico aplicado con y sin los efectos de potencial de impureza se representa en la figura 4. Debido a que el origen de coordenadas se toma en el centro del anillo, y dado que el potencial de confinamiento es simétrico con respecto al mismo puede entenderse por qué, a medida que se aplique el campo eléctrico se presente un corrimiento al rojo del mínimo de las energías en el problema. Teniendo esto en mente, se encuentra la razón por la cual como regla casi general la energía de todos los estados disminuye a medida que crece el campo eléctrico, tal como se muestra en todos los paneles de la figura 4. Nuevamente, como en el caso ya discutido de la figura 2, el potencial de la impureza aumenta el confinamiento de los portadores dando como resultado un corrimiento hacia el rojo de las curvas de energía. Este fenómeno puede ser apreciado al comparar las figuras 4(b) y 4(c) con la figura 4(a). En la figura 4 se aprecian algunos anti-cruces; bien conocidos en la literatura en problemas de campo eléctrico. Tales anti-cruces se entienden como intercambios en la simetría de las funciones de onda de los estados involucrados.

Por otra parte, en la figura 5, se reportan los resultados para los valores esperados de $x$ (debe recordarse que la polarización de la radiación que excita el sistema se ha elegido de forma tal que está escogida en esa dirección) entre diferentes estados inicial y final $\left[M_{f i}=\left\langle\Psi_{f}|x| \Psi_{i}\right\rangle\right]$ en función del campo eléctrico aplicado (a) y de $\alpha_{0}$ (b) para un electrón en un anillo cuántico de GaAs/ $\mathrm{Ga}_{0.7} \mathrm{Al}_{0.3} \mathrm{As}$ con una impureza donadora localizada en $\left(\frac{R_{1}+R_{2}}{2}, 0\right)$. Además, en las gráficas $5(\mathrm{c})$ y $5(\mathrm{~d})$ se presentan, como funciones del campo eléctrico y de $\alpha_{0}$, respectivamente, las energías del estado fundamental, de los dos primeros estados excitados, así como la energía de transición entre el estado fundamental y cada uno de los dos primeros estados excitados. En 5(a), como comportamiento general, se observa que los diferentes elementos de matriz son funciones crecientes del campo eléctrico (excepto $M_{02}$ cuya magnitud es una función decreciente, solo para valores pequeños del campo eléctrico). Esto está en perfecto acuerdo con el incremento del solapamiento de funciones de onda y la fuerte localización de los estados electrónicos alrededor de la posición de la impureza. Otro aspecto a destacar es que, para todo el rango de campo eléctrico considerado, el elemento de matriz $M_{01}$ es idénticamente nulo. Esto se debe a la simetría impar que tiene el primer estado excitado con respecto a la reflexión sobre el eje $x$. 


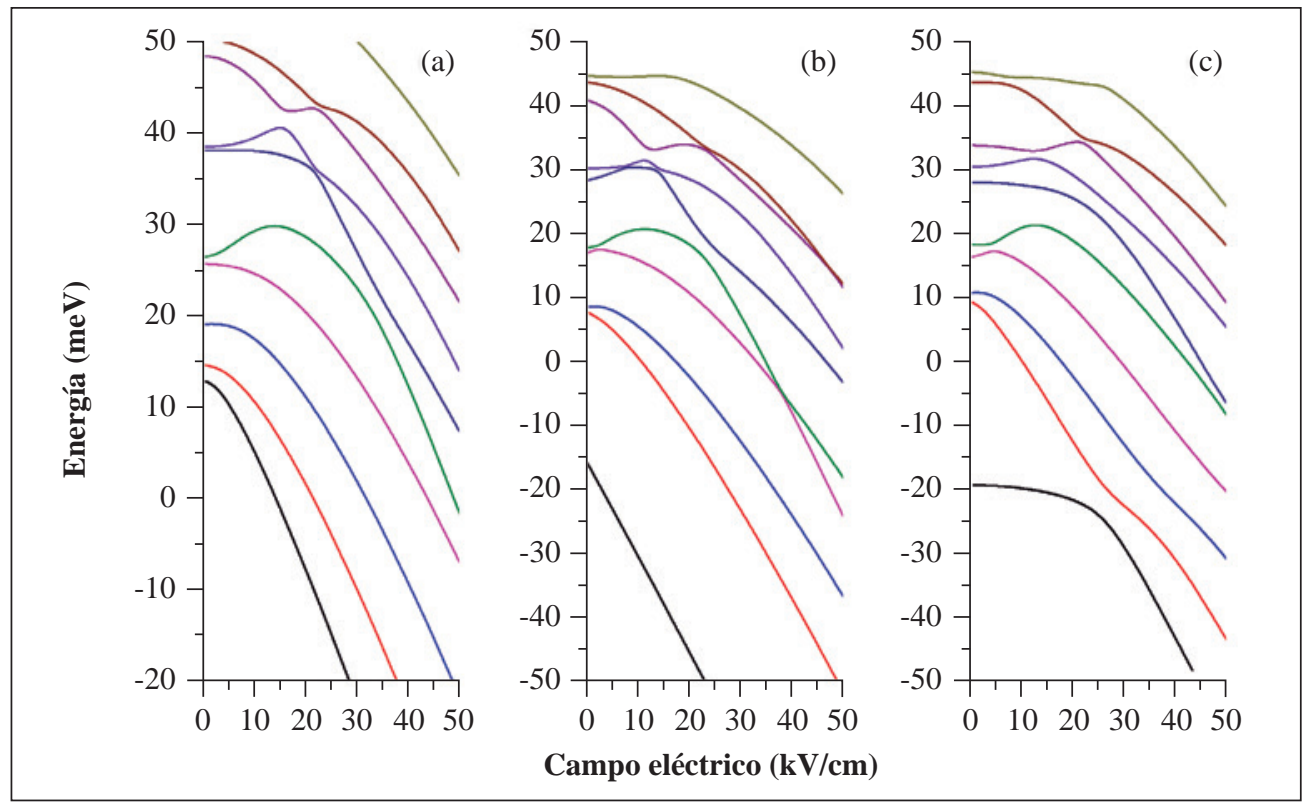

Figura 4. Energía de los primeros estados de un electrón en un anillo cuántico de $\mathrm{GaAs} / \mathrm{Ga}_{0.7} \mathrm{Al}_{0.3} \mathrm{As}$ en función del campo eléctrico aplicado. Los resultados son para $R_{1}=5 \mathrm{~nm}, R_{2}=25 \mathrm{~nm}$ y $\alpha_{0}=5 \mathrm{~nm}$. En (a) no se consideran efectos de impureza mientras que en (b) y (c) la impureza está localizada, respectivamente, en las coordenadas $\left(\frac{R_{1}+R_{2}}{2}, 0\right)$ y $\left(0, \frac{R_{1}+R_{2}}{2}\right)$.

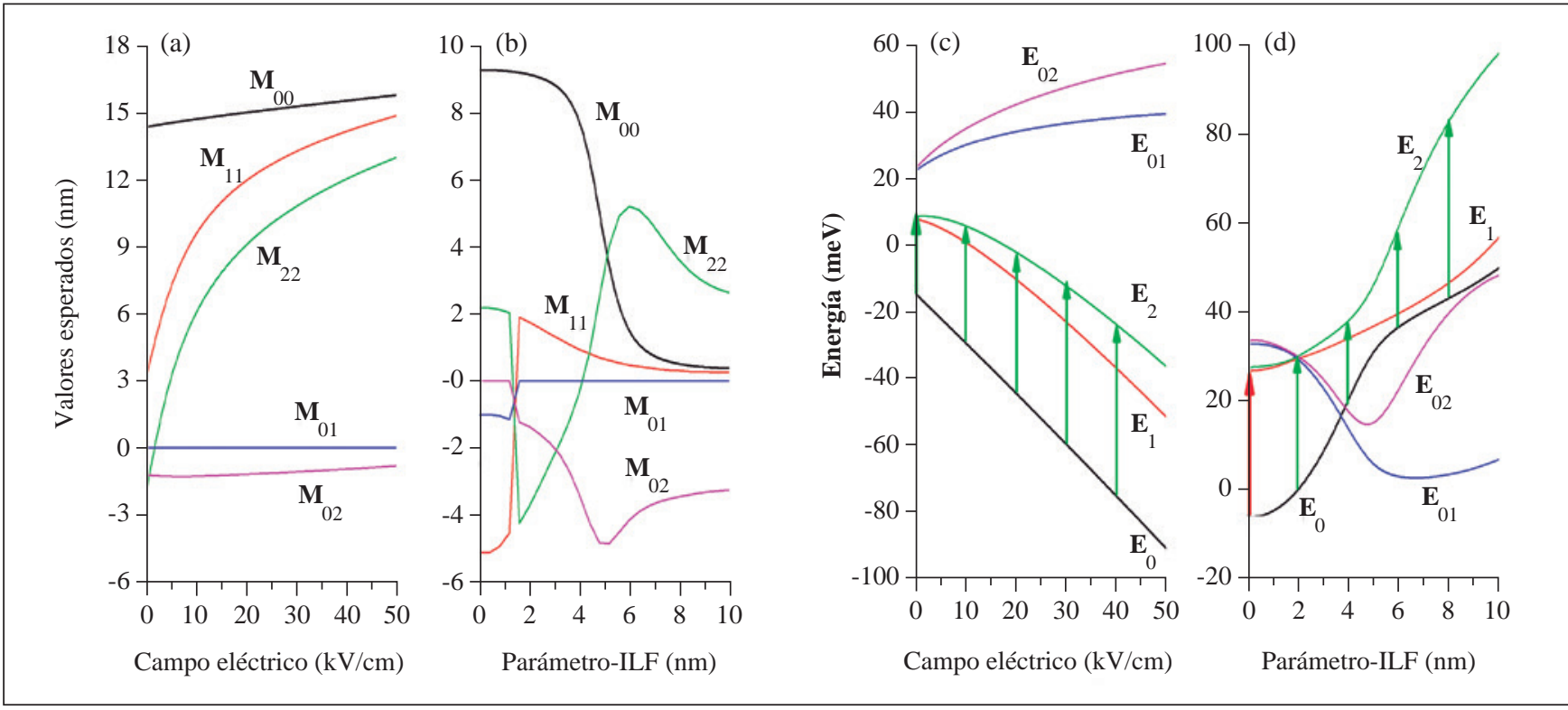

Figura 5. Valores esperados de $x$ entre diferentes estados inicial y final $\left[M_{f i}=\left\langle\Psi_{f}|x| \Psi_{i}\right\rangle\right]$ en función del campo eléctrico aplicado (a) y de $\alpha_{0}$ (b) para un electrón en un anillo cuántico de $\mathrm{GaAs} / \mathrm{Ga}_{0.7} \mathrm{Al}_{0.3} \mathrm{As}$ con una impureza donadora localizada en $\left(\frac{R_{1}+R_{2}}{2}, 0\right)$. En (c) y (d) se presentan en función del campo eléctrico y de $\alpha_{0}$, respectivamente, las energías del estado fundamental, de los dos primeros excitados y la energía de transición entre el fundamental y cada uno de los dos primeros excitados. En (a) y (c) los resultados son para $R_{1}=5 \mathrm{~nm}, R_{2}=$ $25 \mathrm{~nm}$ y $\alpha_{0}=5 \mathrm{~nm}$, y , mientras que en (b) y (d) los resultados son para $R_{1}=5 \mathrm{~nm}, R_{2}=15 \mathrm{~nm}$ y $F=0$. Las flechas verticales en (c) y (d) corresponden a valores escogidos para el cálculo de propiedades ópticas que son reportadas posteriormente.

Si pasamos a analizar los elementos de matriz en función de $\alpha_{0}$, se observan comportamientos mucho más complejos dado los cambios de simetría que sufren las funciones de onda en aquellos puntos donde hay anti-cruces (ver columnas 2 y 3 en la figura 1). Para valores de $\alpha_{0}$ menores que $1.6 \mathrm{~nm}$, es claro que las transiciones se dan desde el estado 
fundamental hacia el primer estado excitado, mientras que para valores mayores, las transiciones son hacia el segundo estado excitado. De los resultados mostrados en las figuras 5(c) y 5(d), se concluye que los picos resonantes o ceros en las estructuras ópticas mostrarán siempre un corrimiento al azul para valores crecientes del campo eléctrico (ver en la figura 5(c) la forma en que crecen las flechas que indican las transiciones para diferentes valores del campo eléctrico). En el caso de la variación asociada al incremento de $\alpha_{0}$, se observa que para valores bajos de dicho parámetro la energía de transición inter-subbanda es decreciente, indicando un corrimiento al rojo en las estructuras de las propiedades ópticas, mientras que para $\alpha_{0}$ mayor que $4 \mathrm{~nm}$, aparece un corrimiento al azul. La combinación de los resultados en las figuras 5(a) y 5(c) o entre 5(b) y 5(d), explicará la magnitud de los picos resonantes en la absorción óptica y de los máximos y mínimos en el índice de refracción, como se verá a continuación.

En la figura 6 hemos representado los resultados del cálculo de los coeficientes de cambio relativo del índice de refracción y de absorción óptica en un anillo cuántico de $\mathrm{GaAs} / \mathrm{Ga}_{0.7} \mathrm{Al}_{0.3} \mathrm{As}$ en función de la energía del fotón incidente; considerando diferentes valores del campo eléctrico 6(a,c) y de $\alpha_{0} 6(\mathrm{~b}, \mathrm{~d})$. Los datos de entrada y las dimensiones de las estructuras son los mismos que se emplearon para obtener la figura 5. Como ya se había predicho en el análisis de dicha figura, aquí se observa un corrimiento constante hacia el azul en ambas propiedades ópticas cuando se incorporan valores crecientes de la intensidad del campo eléctrico estacionario.

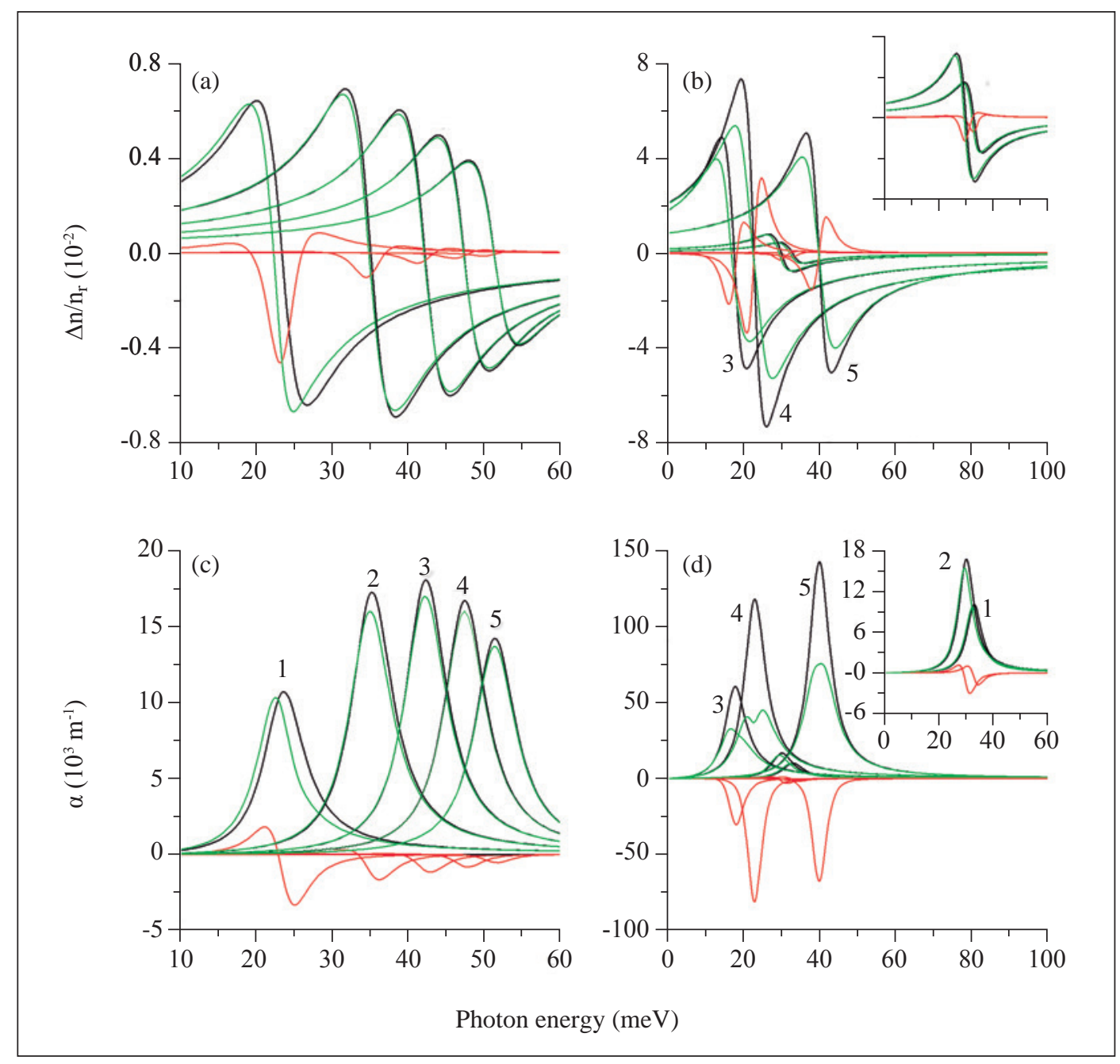

Figura 6. Cambios en el índice de refracción (a,b) y absorción óptica (c,d) en función de la energía de fotón incidente para transiciones intersubbandas para un electrón confinado en un anillo cuántico de $\mathrm{GaAs} / \mathrm{Ga}_{0.7} \mathrm{Al}_{0.3} \mathrm{As}$ con una impureza donadora localizada en $\left(\frac{R_{1}+R_{2}}{2}, 0\right)$. En (a,c) se presentan resultados para cinco valores del campo eléctrico aplicado $(F=0,10,20,30,40 \mathrm{kV} / \mathrm{cm}) \mathrm{con} R_{1}=5 \mathrm{~nm}, R_{2}$ $=25 \mathrm{~nm}$ y $\alpha_{0}=5 \mathrm{~nm}$. En (b,d) se presentan resultados para cinco valores del parámetro de laser $\left(\alpha_{0}=0,2,4,6,8 \mathrm{~nm}\right)$ con $R_{1}=5 \mathrm{~nm}, R_{2}=15$ $n m$ y $F=0$. Las líneas negras, rojas y verdes corresponden, respectivamente, al término de primer orden, el de tercer orden y el valor total bien sea para cambios en índice de refracción como absorción óptica. 
En otro orden de cosas, al observar las líneas 1, 2 y 3 en 6(b) y 6(d) se detecta un corrimiento al rojo en los espectros mientras que de la comparación de las líneas 3, 4 y 5 se concluye la existencia de un corrimiento hacia el azul; tal como se había predicho de la figura 5(d). En cuanto a las figuras $6(\mathrm{a}, \mathrm{c})$ se observa que la magnitud del pico resonante en la absorción y de los máximos y mínimos en los cambios de índice de refracción está gobernada fundamentalmente por el comportamiento de la magnitud del elemento $M_{02}$. Éste, a campos bajos es una función creciente de la intensidad, y luego se convierte en una función decreciente de $F$.

En la figura 6(d) se observa que para valores altos de $\alpha_{0}$, el factor dominante en la amplitud de la respuesta óptica de absorción es la magnitud de la energía de transición intersubbanda mientras que a valores bajos de $\alpha_{0}$ es dominante el factor $M_{01}$. En el caso de cambios en el índice de refracción, figura la $6(\mathrm{~b})$, en el régimen de valores bajos de $\alpha_{0}$ la magnitud de los máximos y mínimos está gobernada por el factor $M_{01}$ mientras que en el caso de valores altos de esta cantidad, tal amplitud está gobernada por $M_{02}$.

\section{Conclusiones}

En este trabajo se ha hecho un estudio teórico de las propiedades ópticas y de impurezas para un electrón confinado en un anillo cuántico de GaAs-(Ga,Al)As sometido a los efectos combinados de campo eléctrico estacionario y radiación de alta intensidad no resonante. Los cálculos se han hecho en la aproximación de masa efectiva haciendo uso de una diagonalización en la cual se ha considerado un conjunto completo de funciones sinusoidales para obtener las auto-funciones y autoenergías del problema. Con las auto-funciones y autoenergías se ha procedido a estudiar la absorción óptica lineal y no lineal y los cambios en el índice de refracción. Los resultados obtenidos sugieren un corrimiento al rojo de las propiedades ópticas en función del campo eléctrico mientras que en el láser no resonante puede inducir efectos combinados de corrimientos al rojo y al azul. De los resultados aquí discutidos se concluye que no es posible hacer predicciones acerca del comportamiento de las propiedades ópticas lineales y no lineales en anillos cuánticos que están sometidos a efectos simultáneos de radiación láser intensa y no resonante, campos eléctricos estacionarios y centros de impurezas. Siempre será necesario recurrir a los cálculos numéricos de las heteroestructuras para así entrar a conocer las diferentes dependencias de la óptica de éstos sistemas, que pueden, en general, resumirse en la ocurrencia de corrimientos hacia más bajas o más altas energías de los picos resonantes -o de los máximos y mínimos- de los coeficientes ópticos estudiados, así como en la variación de las amplitudes de tales respuestas ópticas ante la influencia de los factores externos considerados.

\section{Agradecimientos}

Los autores agradecen a las agencias colombianas: CODIUniversidad de Antioquia (Estrategia de Sostenibilidad 2014-2015 de la Universidad de Antioquia; proyecto "On the way to development of new concept of nanostructure-based $\mathrm{THz}$ laser"; proyecto "Propiedades ópticas de impurezas, excitones y moléculas en puntos cuánticos autoensamblados"), Facultad de Ciencias Exactas y Naturales-Universidad de Antioquia (CAD y ALM-proyecto de dedicación exclusiva 2014-2015), El Patrimonio Autónomo Fondo Nacional de Financiamiento para la Ciencia, la Tecnología y la Innovación, Francisco José de Caldas.

\section{Conflicto de intereses}

Los autores declaran que no tienen conflicto de intereses.

\section{Referencias}

Aharonov Y., Bohm D., 1959. Significance of Electromagnetic Potentials in the Quantum Theory. Physical Review 115: 485-491.

Aichinger M., Chin S. A., Krotscheck E., Räsänen E. 2006. Effects of geometry and impurities on quantum rings in magnetic fields. Phys. Rev. B 73: 195310-195310-8

Asmar N. G., Markelz A. G., Gwinn E .G., Cerne J., Sherwin M. S., Campman K. L., Hopkins P. F., Gossard A. C. 1995. Phys. Rev. B 51: 18041-18044.

Assaid E., Aydi M., Feddi E., and Dujardin F. 2008. Exact analytical solutions for shallow impurity states in symmetrical paraboloidal and hemiparaboloidal quantum dots. Cent. Eur. J. Phys. 6: 97-104.

Barseghyan M. G., Restrepo R. L., Mora-Ramos M. E., Kirakosyan A. A., and Duque C. A. 2012. Donor impurity-related linear and nonlinear intraband optical absorption coefficients in quantum ring: effects of applied electric field and hydrostatic pressure. Nanoscale Research Letters 7: 538.

Bayer M., Hawrylak P., Hinzer K., Fafard S., Korkusinski M., Wasilewski Z. R., Stern O., Forchel A. 2001. Coupling and Entangling of Quantum States in Quantum Dot Molecules. Science 291: 451-453.

Bruno-Alfonso A., Latgé A. 2000. Semiconductor quantum rings: Shallow-donor levels. Phys. Rev. B 61: 15887-15894.

Culchac F. J., Porras-Montenegro N., Latgé A. 2008. GaAs$(\mathrm{Ga}, \mathrm{Al}) \mathrm{As}$ double quantum rings: confinement and magnetic field effects. J. Phys.: Condens. Matter 20: 285215-1-285215-6.

Duque C. A., Kasapoglu E., Sakiroglu S., Sari H., and Sökmen I. 2010. Intense laser effects on donor impurity in a cylindrical single and vertically coupled quantum dots under combined effects of hydrostatic pressure and applied electric field. Applied Surface Science 256: 7406-7413.

Duque C. A., Mora-Ramos M. E, Kasapoglu E., Sari H., Sokmen I. 2011. Intense laser field effect on impurity states in a semiconductor quantum well: transition from the single to double quantum well potential. Eur. Phys. J. B 81: 441-449. 
Filikhin I., Suslov V. M., Vlahovic B. 2006. Electron spectral properties of the InAs/GaAs quantum ring Physica E 33: 349-354.

Fulla M. R., Marín J. H., Gutiérrez W., Duque C. A., and MoraRamos M. E. 2014. $\mathrm{D}_{2}^{+}$molecular complex in ring-like nanostructures: hydrostatic pressure and electro-magnetic field effects. Acta Physica Polonica 125: 241-244.

Garcia J. M., Medeiros-Ribeiro G., Schmidt K., Ngo T., Feng J. L., Lorke A., Kotthaus J., Petroff P. M. 1997. Intermixing and shape changes during the formation of InAs self-assembled quantum dots. Applied Physics Letters 71: 2014-2016.

Harutyunyan V. A. 2009. Semiconductor nanocylindrical heterolayer in a radial electrostatic field: electronic spectrum and optical properties. Applied Surface Science 256: 455 - 459 .

Harutyunyan V. A. 2011. Semiconductor nanotube in strong electrostatic field. Journal of Applied Physics 109: 0143251-014325-8.

Kasapoglu E., Duque C. A., Sari H., Sokmen I. 2011. Intense laser field effects on the linear and nonlinear intersubband optical properties of a semi-parabolic quantum well. European Physical Journal B 82: 13-17.

Kleemans N. A. J. M., Blokland J. H., Taboada A. G., van Genuchten H. C. M., Bozkurt M., Fomin V. M., Gladilin V. N., Granados D., Garcia J. M., Christianen P. C. M., Maan J. C., Devreese J. T., Koenraad P. M. 2009. Excitonic behavior in self-assembled InAs/GaAs quantum rings in high magnetic fields. Phys. Rev. B 80: 155318-155318-4.
Lorke A., Luyken R. J., Govorov A. O., Kotthaus J. P., Garcia J. M., Petroff P.M. 2000. Spectroscopy of Nanoscopic Semiconductor Rings. Physical Review Letters 84: 22232226.

Martínez-Orozco J. C., Mora-Ramos M. E., and Duque C. A. 2012. The nonlinear optical absorption and corrections to the refractive index in a GaAs n-type delta-doped field effect transistor under hydrostatic pressure. Physica Status Solidi (b) 249: 146-152.

Monozon B. S., Schmelcher P. 2003. Impurity center in a semiconductor quantum ring in the presence of crossed magnetic and electric fields. Phys. Rev. B67: 045203045203-14

Mora-Ramos M. E., Duque C. A., Kasapoglu E., Sari H., Sokmen I. 2013. Electron-related nonlinearities in GaAsGa1-xAlxAs double quantum wells under the effects of intense laser field and applied electric field. J. Lumin. 135: 301-311.

Peyghambarian N., Koch S. W., Lindberg M., Fluegel B., Joffre M. 1989. Dynamic Stark effect of exciton and continuum states in CdS. Phys. Rev. Lett. 62: 1185-1188.

Radu A., Kirakosyan A. A., Laroze D., Baghramyan H. M., and Barseghyan M. G. 2014. Electronic and intraband optical properties of single quantum rings under intense laser field radiation. Journal of Applied Physics 116: 093101.

Xia J-B. and Fan W-J. 1989. Electronic structures of superlattices under in-plane magnetic field. Phys. Rev. B40: 8508-8515. 